\title{
Proposed Ontology for Cognitive Radar Systems
}

\author{
Colin Horne*, Matthew Ritchie, Hugh Griffiths \\ Department of Electronic and Electrical Engineering, University College London, London, UK \\ *uceehor@ucl.ac.uk
}

\begin{abstract}
Cognitive radar is a rapidly developing area of research with many opportunities for innovation. A significant obstacle to development in this discipline is the absence of a common understanding of what constitutes a cognitive radar. The proposition in this article is that radar systems should not classed as cognitive, or not cognitive, but should be graded by the degree of cognition exhibited. We introduce a new taxonomy framework for cognitive radar against which research, experimental and production systems can be benchmarked, enabling clear communication regarding the level of cognition being discussed.
\end{abstract}

\section{Introduction}

The term 'cognition' emerged into the vocabulary of the radar literature in 1990 [1], progressing to the phrase 'cognitive radar' (CR) in the early 2000s [2]. At this early stage the concepts of learning, knowledge and feedback between receiver and transmitter were established as necessary components for a radar to be described as cognitive. CR became fully established in Haykin's 2006 paper [3]. The relationship between CR and the early work in cybernetics, CRs principal components, and potential benefits are explored in [4]. One important benefit CR might offer is the mitigation of negative performance effects caused by reduced bandwidth availability for radar operations. This bandwidth reduction is due to the growth in demand for RF spectrum from competing user communities, and the financial dividend available to governments from fulfilling that demand. In [5,6] the UK Department for Culture Media \& Sport states that: 'Spectrum is hugely valuable. In economic terms it is already worth over $£ 50$ bn a year to the UK economy', and '.. at least $500 \mathrm{MHz}$ of public sector spectrum holdings below $5 \mathrm{GHz}$ would be released by 2020'. High spectrum value is a global phenomenon, as evidenced by the greater than $\$ 41$ billion raised by the 2014/2015 AWS-3 auction, conducted by the US Federal Communications Commission, selling just 65 $\mathrm{MHz}$ of bandwidth [7].

The economic advantage to be gained from technologies such as CR providing reduced instantaneous spectral occupancy by radar systems is therefore clear. In addition to the economic considerations, attention must also be given to the increasing complexity and hostility encountered in radar system operation, due to increased spectral congestion, and the use of more effective electronic countermeasures. These factors require a new approach to radar system design.

Spectrum occupancy and associated waveform design opportunities are well represented in the CR literature. The possible gains to be made by employing cognition in radar systems may be derived by the adaptive control of a wide range of other radar system parameters. Examples include the selection of transmission power, polarisation, Pulse Repetition Frequency (PRF), dwell time on target, scenario geometry, and the choice of illuminator of opportunity in passive radar.
The technological advances which have enabled the expansion of mobile communications availability, and with it, the increased requirements for RF bandwidth, may also offer the tools for the solution to the challenges in the CR research domain. Modern levels of computing power, innovative algorithm developments, advances in digital arbitrary waveform generation and RF transmission, can together provide the hardware and software capabilities necessary to design and build CR.

Based on the numbers of recent publications appearing in the literature, see section $2, \mathrm{CR}$ will continue to receive the levels of attention it has attained thus far, and future radar research will likely be increasingly targeted towards adaptivity and cognition. The domain will likely see a convergence of the theoretic concepts of cognition and realtime experimental capability of research RF systems, taking CR to a higher Technology Readiness Level (TRL) [8].

Despite the strong interest in CR, there is currently no measure which allows for the classification of the cognitive capabilities of a radar system to provide all stakeholders with a common understanding of the cognitive features available.

The objective of this article is to develop a systematic ontology that allows for the classification of the full range of radar systems including non-adaptive, adaptive, fully adaptive radar (FAR), and CR, all on a single scale. Thus enabling a common understanding of the capabilities of these diverse systems, and the techniques that are described as cognitive.

Classification schemes can be seen to be applied in many technical areas as a means of distinguishing the capabilities of one example of a given system from another. By way of illustration two examples of schemes loosely related to CR classification are briefly discussed next.

\section{A. UnManned Systems/Unmanned Marine} Systems (UMS): Multiple classification schemes are used by different organisations to define the levels of autonomy exhibited by a given system. The European Defence Agency (EDA) employ a 6 level system [9] where 0 indicates a 'Human on board', through to 5 which means the vehicle is totally autonomous. The US National Institute of Standards and Technology use the Autonomy Levels For Unmanned Systems (ALFUS) framework [10] which considers overall mission complexity in terms of orthogonal factors, namely, 
mission complexity, environmental complexity and human independence. A 0 to 10 scale is applied to each axis, and the values combined to produce an overall score. The ALFUS document also includes descriptions of other similar scales used by NASA and the Army Science Board.

B. Measuring the Level of Consciousness in Artificial Agents: 'One of the first conclusions that one comes to when studying consciousness is that it is a graded phenomenon.' This quote is taken from [11] which describes a framework for analysing the relationship between consciousness and associated cognitive functionality in artificial agents, ConsScale. This scale uses several of the attributes we need to assess in classifying CR, such as perception, adaptation, attention and learning. Consciousness also adds emotion, imagination and self-awareness to the synthesis. ConsScale is concerned with higher order processes, that of machine consciousness, which is beyond what we are considering in this work, with FAR and CR fitting into the narrow region of ConsScale levels 2 (Reactive) to 5 (Executive). ConsScale level 6 is described as 'Emotional' - there is certainly no place for an emotional response from a cognitive radar!

The remainder of this paper is structured as follows: Section 2 describes the characteristic features of CR, and provides the motivation for the requirement for a $C R$ classification scheme, followed in Section 3 by a description of the proposed classification scheme. Finally, Section 4 provides a summary and conclusions.

\section{The Motivation for a Cognitive Radar Classification Scheme}

Over 1000 (June 2018, Full Text \& Metadata search) publications on IEEEXplore currently include the phrase 'cognitive radar', over 100 being added in 2018 alone, demonstrating the significant and growing interest in the domain of CR. However, when assessing CR research there is no metric available for measuring the level of cognition exhibited to substantiate the designation of CR. Another issue which is often ignored when work is described as CR, is that a radar system is unlikely to be either purely cognitive in all aspects, or totally non-cognitive. As radars are complex systems, certain aspects of the system may be cognitive to some degree, while other aspects are not. Therefore it is also important to consider, and to specify, which aspects of a radar system are being described as cognitive.

The focus of this work is on the classification of synthetic, or human-independent, fully adaptive/cognitive systems, but attention is also afforded to the 'edge cases' where human involvement is present, and to radar systems which exhibit only 'adaptive-on-receive' behaviour, hence not being what would be termed 'fully adaptive'.

This section provides a summary of the nature of adaptive radar systems, including adaptive-on-receive, FAR, and CR. The purpose of this review is to provide the context for the proposal of the ontology covering the range of radar systems under investigation.

We begin by providing a brief overview of CR, with particular regard to the agreement of the attributes and characteristics necessary for cognition to exist. The initial focus is on the two researchers who have defined the landscape of CR. This is followed by a more general review of CR literature. We subsequently compare the definitions of various adaptive radar 'flavours'.

\subsection{Cognitive radar}

It is recognised in [3] that $\mathrm{CR}$, and the environment within which it operates, constitutes a closed-loop feedback system. An action, possibly a signal transmission, is instigated by the CR. This signal interacts with the environment, including clutter and targets of interest, producing returns, which are subsequently collected by the radar receiver. The backscattered returns are analysed by the signal processing system to extract the salient features of the scene, which may be fused with a priori knowledge and information from other sensors, both on-board and remote. The aim is to create an understanding of the critical components of the environment. From this 'perception' the system is able to reason as to the appropriate next action to take. This repetitive process, termed the 'cognitive signal processing cycle', is a radar centric version of the human cognitive principles described in [12], which in the context of human cognition, is called the 'perception-action cycle' (PAC). The PAC, modified from a version by Haykin [13], is illustrated in Fig. 1. The PAC, along with the four other characteristics of memory (or learning), attention, intelligence and language [12], are the cornerstones of current cognitive science in terms of characterising the necessary components of cognition, and will play a central role in the ontology discussed later in this article.

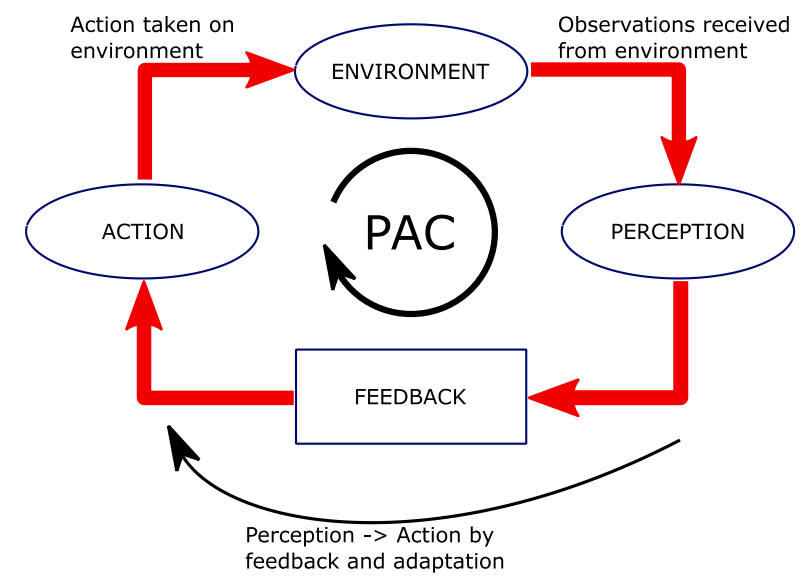

Fig. 1: The Perception-Action Cycle

Haykin extends the application of cognition to networked radar scenarios [14], which in addition to the internal operation of the PAC, also includes a central hub fusing information from multiple sensors. The aim being to make more informed choices of future radar actions across the entire network. Other researchers working on knowledge based intelligent multisensory radar networks [15] do not employ the receiver to transmitter feedback described by Haykin.

Haykin has continued to work on various aspects of CR including waveform design [13], tracking [16,17], cognitive control [18,19] and cognitive radar networks [20,21].

Guerci, with others, has long advocated the use of adaptive waveform generation for improving radar performance in terms of target detection and identification 
[22,23]. Guerci has also made significant contributions to our understanding of what constitutes $\mathrm{CR}$, by linking cognitive and knowledge based radar systems research through the KASSPER [24] programme, and adaptive radar architecture research [25]. The Knowledge Aided, Fully Adaptive Cognitive Architecture is shown in Fig. 2. The vision expressed in these works emphasises the knowledge element of future radar systems. But central to the architecture are the same components defined by Fuster [12] and Haykin, the PAC, which provides a framework for sensing and action, memory, in the form of learning and knowledge, attention as signified by a tasking process focusing resources on items of interest, and intelligence, implemented by mission computers and CoFar co-processors.

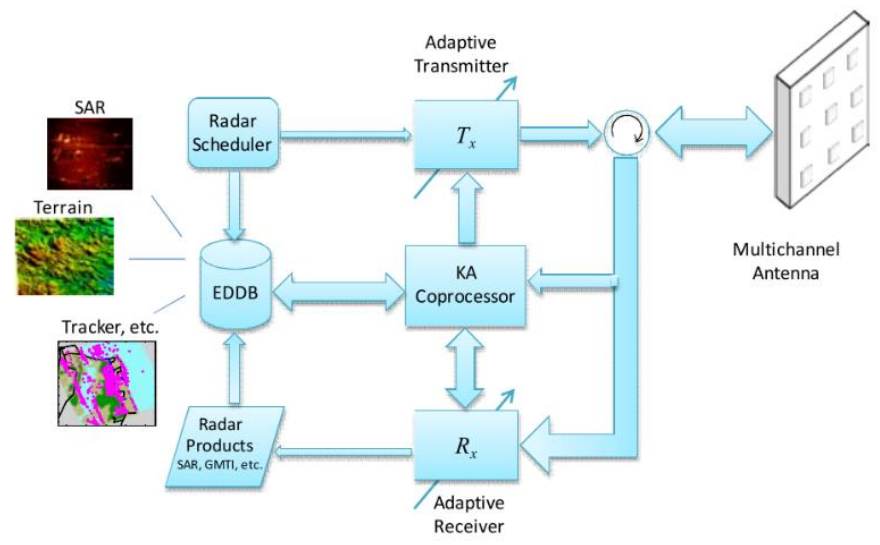

Fig. 2: Knowledge Aided, Fully Adaptive Cognitive Architecture [26]

The inspiration for CR is rooted in the natural world. It has long been known that echo locating bats can adjust the ultrasonic signals they transmit. In 1965 it was shown [27] that bats can learn to discriminate between edible and inedible flying objects. Initially the bats would catch both types of object with equal success. After training, attention was focused on the more tasty variety. This result confirms that bats are capable of learning from the environment, and change behaviour based on that learning. Results reported in 2003 show that bats discriminate between plants which had not yet been visited, and are therefore a good source of nectar, and those that had already been visited [28]. In this work bats also learned to discriminate between replica plant features with different spectral responses, and focused their attention on the features which indicate a reward is available. Vespe et al. [29] investigate the waveforms employed by echolocating bats, and show the complex dynamic nature of their transmitted signals in terms of frequency, bandwidth and modulations. The evolution of some plant species such that their echo location signature in some way enables bats to identify the plant as a good source of food is an example of exploitation of third party transmissions [30]. Echoic flow [31] is a function seen in bats which contributes to successful navigation, and could provide inspiration for synthetic cognitive navigation and intercept point planning.

Dolphins and whales also employ echolocation techniques [32]. Echolocating porpoises exhibit the ability to reduce the beamwidth of their sonic transmissions during the hunt terminal phase, to prevent prey from escaping by moving outside of the hunters field of view [33]. Unique human individuals also use echolocation, generating sounds by tongue clicking $[34,35]$. For more details on biologically inspired radar and sonar systems see [36], and references therein.

As was highlighted in the introduction, spectral occupancy and adaptive waveform design, or waveform diversity [37], are particularly prevalent themes researched under the CR heading. Wicks [38], and Griffiths et al. [39], outline the spectrum crowding issues, and how cognition might be applied to address the problem. Along with the technical aspects, regulatory perspectives must also be considered [40]. As long ago as 1967/69, DeLong and Hofstetter [41, 42] examined the iterative design of waveforms for optimum target detection in thermal noise and clutter. Stinco et al. [43] discuss sensing of the spectral environment, and how spectrum sharing can achieve minimal interference between users, using cognitive techniques. Goodman [44] describes a framework for implementing closed-loop waveform design. A cognitive processor is described [45] capable of learning the spectral content of the environment for use in creating an appropriate notched waveform for transmission, while the same authors consider the impact of the notched waveforms to beamforming in wideband phased array radars [46]. Aubry et al. employ information from a radio environmental map to provide constraints for waveform design in crowded environments [47]. In [48] the performance of waveforms created by proposed design techniques are assessed in terms of the tradeoffs between competing requirements of signal to interference plus noise ratio, waveform features, and radiated energy. Aubrey et al. [49] investigate both transmit signal and receive filter design in high clutter environments. Blunt and Mokole [50] provide a detailed review of waveform diversity techniques and challenges, including comprehensive references.

Adaptive waveform design for matched illumination is investigated for the purposes of target detection and identification. Gjessing $[51,52]$ worked extensively to advance this subject. Matched illumination of ground targets is investigated [53] using models of vehicles in experimental trials. The use of SNR and mutual information in waveform design for target recognition are examined in [54,55], and matched illumination waveforms and sequential hypothesis testing in [56].

CR networks have continued to receive attention and are examined in $[57,58]$ investigating suitable network architectures, and $[59,60]$ for target tracking. The application of beam steering in CR networks is discussed in [61]. Sensor management is an important consideration in $\mathrm{CR}$, examined in $[62,63]$, and [64] considers the control of a multistatic multifunction radar network consisting of both active and passive radar nodes.

An area of research which has the potential to inform our understanding of CR is in the creation of frameworks and architectures in which to instantiate CR systems. Bell et al. [65] describe a framework for a fully adaptive radar which demonstrates the advantage to be gained by active control of radar parameters. The framework is subsequently implemented on a general purpose hardware platform, specifically designed for FAR and CR research [66]. Oechslin et al. [67] describe a CR testbed, and compare its characteristics with those of the framework described in [66].

Exponents of HF radar argue that this technology is an existing example of CR. This is justified on the basis that dynamic ionospheric and spectral occupancy conditions 
require constant monitoring and interpretation to enable the selection of appropriate radar operating parameters. In 1986 expert systems were applied to HR radar control [68]. Lu and Chen [69] describe the merging of HF radar with CR to create systems less dependent on highly skilled operators. Cognitive waveform parameter selection on the basis of a priori information, and external ionosphere measurements is described in [70]. Holdsworth discusses performance assessment in cognitive over-the-horizon radar using synthetic targets [71].

Wicks [72] foresees a future in which the integration of cutting edge technologies such as knowledge-based signal processing, robotics, wireless networking and waveform diversity, combine to create systems with extraordinary capabilities. A truly ambitious aim in 2003.

Along with the potential gains of $\mathrm{CR}$, we should also consider the problems which might be encountered as we progress towards practical CR. Greenspan [73] highlights several 'potential pitfalls' where caution should be exercised, from the reliability of knowledge sources, to the extent of training times required for learning machines, and the potential for learning stagnation. The legal implications of inappropriate actions taken by intelligent machines such as CR must also be carefully considered.

The review provides the foundations of our current understanding of CR. The body of literature covering pre-CR, and the current state-of-the-art, provides a broad view of the subject of CR, and illustrates the wide-ranging nature of the subject. However, it has not changed, added to, or clarified the true nature of CR significantly. This lack of progress is highlighted in [74] in which the author states (2016) 'There is still no exact definition in the community, on what discriminates an adaptive from a cognitive radar'.

There is general agreement among the CR research community concerning the elements which must be present in a radar system for it to be classed as cognitive, these being unchanged since Haykin's early work on CR. However, the cognitive abilities of specific CR implementations is still unclear.

\subsection{Adaptive radar (AR), cognitive radar (CR), fully adaptive radar (FAR) and intelligent radar (IR)}

In this subsection, we address the definitions of adaptive radar variants, and how the various adaptive radar terms apply in the remainder of this work.

'adaptive radar: A radar system that adapts its processing and control to improve achievement of a desired function' [75]. In this article adaptive radar represents the container for all forms of adaptivity.

'cognitive radar: A radar system that in some sense displays intelligence, adapting its operation and its processing in response to a changing environment and target scene. In comparison to adaptive radar, cognitive radar learns to adapt operating parameters as well as processing parameters and may do so over extended time periods' [75].

This definition has been debated at length, in particular, by members of the NATO SET 227 panel on Cognitive Radar, and represents the best high level definition currently available. The CR definition extends that of AR to specifically include the characteristics of intelligence and learning.
Even within the relatively small community engaged in CR research there can be significantly diverse views on what constitutes a CR. For example, the question as to if a standard air traffic control (ATC) radar system can be classified as a CR? Baker and Smith [76] argue that, from a systems point of view, ATC can be seen as a CR. This view is based on the understanding that the radar acts as a sensor, providing data or information to the human controllers. The controllers interpret the information to produce an understanding of the scene, the perception, and issue flight commands, the action, to the pilots, who in turn implement the action, and consequently cause the environment to change by potentially altering the course and position of their aircraft. Therefore the system can be regarded as cognitive by virtue of the human-in-the-loop providing the cognitive capability. It additionally meets the requirements of $\mathrm{CR}$ as specified in section 2.1 .

In this work we use $\mathrm{CR}$ to represent machine implemented 'synthetic cognition', as opposed to human included cognition.

The title 'cognitive radar', is a basis of heated discussion, with alternative labels such as 'fully adaptive radar' being suggested. For the purposes of clarity, in this work the term FAR is used to indicate a radar system which is adaptive in more than precisely the receiver chain. It must include the PAC, and have the ability to modify some aspect of its own behaviour such that the environment being sensed, or at least the response created from the environment, is in some way changed by its actions. CR is seen as the logical extension of FAR, providing the recognisable traits of cognition. The transition between FAR and CR is regarded as a smooth evolution rather than separate characteristics. Additionally we use the term Intelligent Radar (IR) as a container to encompass all forms of FAR and CR.

There is a pressing need to define an ontology which encompasses the range of systems, from those which show little or no cognitive capabilities, covering systems which exhibit cognition by virtue of a human in-the-loop, through to the long term goal of $\mathrm{CR}$ research, the fully synthetic cognitive systems which display, what might be termed, human levels of cognition or beyond, but implemented purely by machines. Beyond human levels of cognition might be understood to be the cognitive capability levels of human cognition without the limitations of processing speed, or the reliability issues, which might be suffered with human fatigue. In contrast, it could also be taken to be learning, decision making, and long term planning capabilities far beyond that of the human mind. By creating a classification system, the domain of $\mathrm{CR}$ can develop further by clarifying the contributions of new CR research.

\section{Proposed classification scheme}

The principal question to be addressed is how to partition the range of possible radar systems such that a clear understanding of the capabilities of systems can be established. There are numerous approaches to describing radar systems [77]. At the top level this might be distinguishing by Monostatic vs Multi-static, Primary vs Secondary, Ground based vs. Airborne vs. Maritime. The purpose of the scheme investigated in this article is to categorise in terms of the level of system adaptivity. The initial branch separates non-adaptive and adaptive radars. The 
tree structure shown in Fig. 3 illustrates the taxonomy proposed.

FAR conventionally means adaptivity on transmit and receive, with feedback taking place between the two, such that the system adapts based on the sensed environment. However, the FAR definition should also embrace systems which have no control of the transmitter, but are still adaptive in more degrees of freedom then receive only. This includes passive bistatic radar (PBR), where cognition could be exhibited in the adaptive selection of available illuminators of opportunity [78] based on previous observations, geometry or channel occupancy. Another example being mobile systems adaptively manoeuvring such that their location provides beneficial performance based on the scenario geometry. This generalisation expands the range of systems for which the fully adaptive and cognitive terms, and therefore the classification scheme, may apply.

It could be argued that fully adaptive and cognitive radars should appear on separate branches of the taxonomy. However, this separation would require very clear criteria differentiating between the two branch classifications. The very issue this article is addressing.

The green (solid colour) portion of the taxonomy illustrated in Fig. 3 shows the well understood non-adaptive, human-in-the-loop adaptive, and the existing synthetic adaptive-on-receive flavours of radar systems. Examples of the human-in-the-loop are simple threshold selections, possibly based on visual information presented on a PPI display, radar mode selections, for example surveillance or tracking modes, and the more sophisticated air traffic control application as discussed in Section 2.2. The adaptive on receive systems encompass applications from the relatively simple constant false alarm rate mechanisms based on the assumed statistics of the observed signals, to the complex, processing intensive, space-time adaptive processing systems.

The blue (hatched) region shows the space where further refinement of our understanding of IR is required. The degree of cognition exhibited is seen at the bottom level of the IR branch, increasing from left to right. The term 'minimally fully adaptive' (MFA) representing the lowest level of fully adaptive systems envisaged, and cognitive the most 'intelligent'. We require a mechanism to differentiate between the base level categories. For this we return to the five characteristics described by Fuster [12] necessary for cognition. Firstly we consider to what degree each contributes to the level of cognitive behaviour exhibited by an IR. In this work we consider the characteristics of the PAC, attention and language as all being profoundly necessary in any IR system. These characteristics must be included to the 'necessary degree', in all systems claiming some level of IR capability. The 'necessary degree' constraint means sufficient to provide the capability required by all other aspects of the system. How is this constraint justified? Consider the PAC. The PAC is the facility of the adaptive system which implements its closed-loop feedback nature, and therefore must exist for adaptation to the environment to exist. Attention enables the adaptive system to focus its resources on some critical aspects of the observed scene, without which the objectives of the sensor would be unclear. It might be argued that attention has different levels of capability, focussing attention on multiple items of interest in the multiple target tracking scenario. The view taken here, is that this scenario corresponds to multiple attention objects, each carrying out the attention function, rather than different levels of attention. The language characteristic can be viewed as the ability to store, use and disseminate information from the system, without which there is no recognised cognition.

Therefore, the characteristics which will differentiate between lower level fully adaptive, higher functioning fully adaptive, and cognitive radar will be those of memory and intelligence. The challenge is how to define the nature of these two entities in ways which can differentiate the range of IR definitions.

Memory is viewed as the ability to learn and access knowledge, using language to store and retrieve data. It will be thought of functioning at the following levels : i) as a fixed internal knowledge base, ii) as a dynamic knowledge base updated by an external agent, and iii) as an on-line learning-

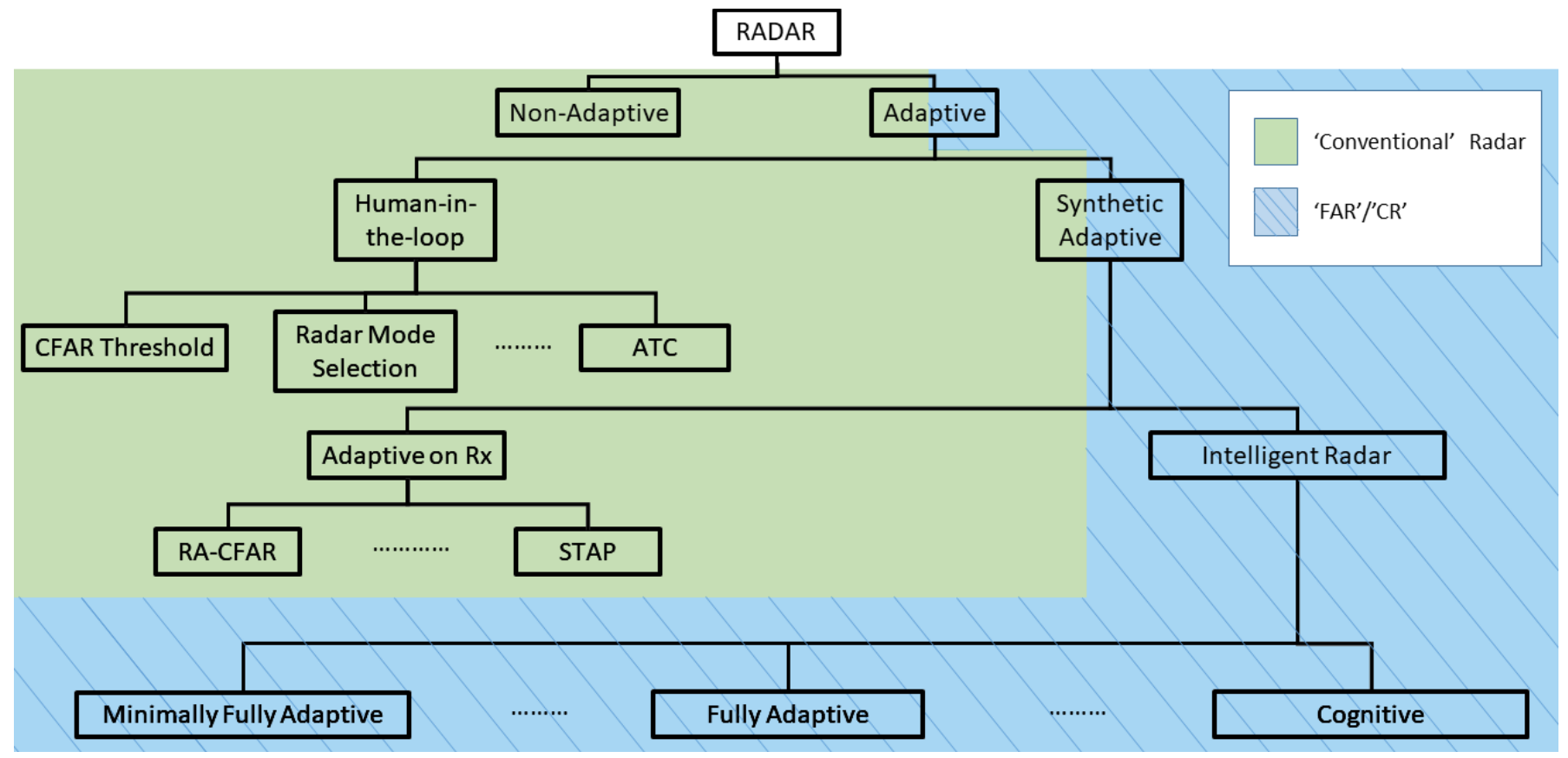

Fig. 3: Radar Taxonomy - Adaptive Radar Centric 
capable system. Each level will be sub-divided further as necessary.

Intelligence as a characteristic is harder to describe. Consider Vernon's [79] characterisation of 'agents with cognitive abilities', '.. systems which exhibit adaptive, anticipatory, and purposive goal-directed behaviour'. The adaptive element has already been included in our understanding, but can the remaining elements of Vernon's statement be taken as surrogates for intelligence?

Vernon goes on to state 'Cognition implies an ability to understand how things might possibly be, not just now but at some future time, and to take this into consideration when determining how to act.'

This statement corresponds to this authors' view, that cognition is not only concerned with the immediate future, a myopic viewpoint, but instead, should include the ability to anticipate $[80,81]$ and plan future actions, over an extended period, acting in a non-miopic way based on the expected evolution of the scenario.

These arguments lead us to the proposal that two features should replace the 'intelligence' characteristic in the new classification scheme. The features are: i) the decision making mechanism, and ii) the degree of non-myopic behaviour exhibited.

i) High quality decision making is central to an intelligent system. It is based on the perception gained from all the possible sensors, on the fixed knowledge base, on external or experiential knowledge contained in memory. Decision processing can itself take on a range of complexity, from the implementation of simple rules, the use of heuristics, which attempt to provide a more flexible response to that of the simple rule base, but without excessive computational loading, or a full optimisation process which ensures the optimal solution given all the available information. Tweedale [82] provides a useful review of decision making in artificial system. Tweedale argues that humans experience new situations within some recognisable context due to previous experience, so do not approach new situations without any information. This suggests cognitive decision making is tightly linked to the learned memory of previous events, and artificial systems should maintain similar links. The decision making mechanisms form the basis for one dimension of the proposed classification system. As systems take on more complex decision making functionality, the higher the processing load, but with increased probability of successful task outcome.

ii) A system which can plan only one step into the future is adaptive, and can be intelligent. For the purposes of this taxonomy, as a system moves from myopic to nonmyopic functionality, so the classification moves from adaptive to cognitive. So, for a radar to be regarded as fully cognitive it should in some way balance short term gains against longer term benefits of a seemingly non-optimum short term action. In the extreme, for a system to be classed as fully cognitive, the anticipation must extend to the timeframe of the wider mission objectives.

Timescale constraints and computational processing load can also be considered in cognitive assessment. Kyllonen and $\mathrm{Zu}$ [83] suggest that, in the human context, response time can be used as a measure of cognitive ability. Does a system which converges upon a solution more quickly as compared with a competitor show higher cognition? Alternatively, should a system which results in a more accurate solution in the available time be considered 'more cognitive'? For the purposes of classifying IR in this article, we consider this computational complexity as being an implementation issue, and not part of the classification. Although the computational load should be considered when designing IR, it will not contribute to the cognitive ability definition.

The classification scheme proposed has three elements as illustrated in Fig. 4. The 3-dimensional cube represents the space occupied by synthetic IR systems. It can be seen that the 'minimally fully adaptive radar' sits at the bottom left of the space, taking its character from the lower ends of each dimension. The minimally fully adaptive radar exhibits a myopic nature, with fixed decision rules and fixed memory structure. It must, however, include the PAC, attention and language capabilities. The 'fully cognitive radar' sits at the opposite extreme, exhibiting high levels of optimised decision making, learning and non-myopic planning. The three dimensions contribute equally to the final cognitive classification, although differential weighting could be applied if the characteristics are found to be of non-equal importance. Any such weighting must be specified such that the relative importance of each dimension value is understood by the whole community.

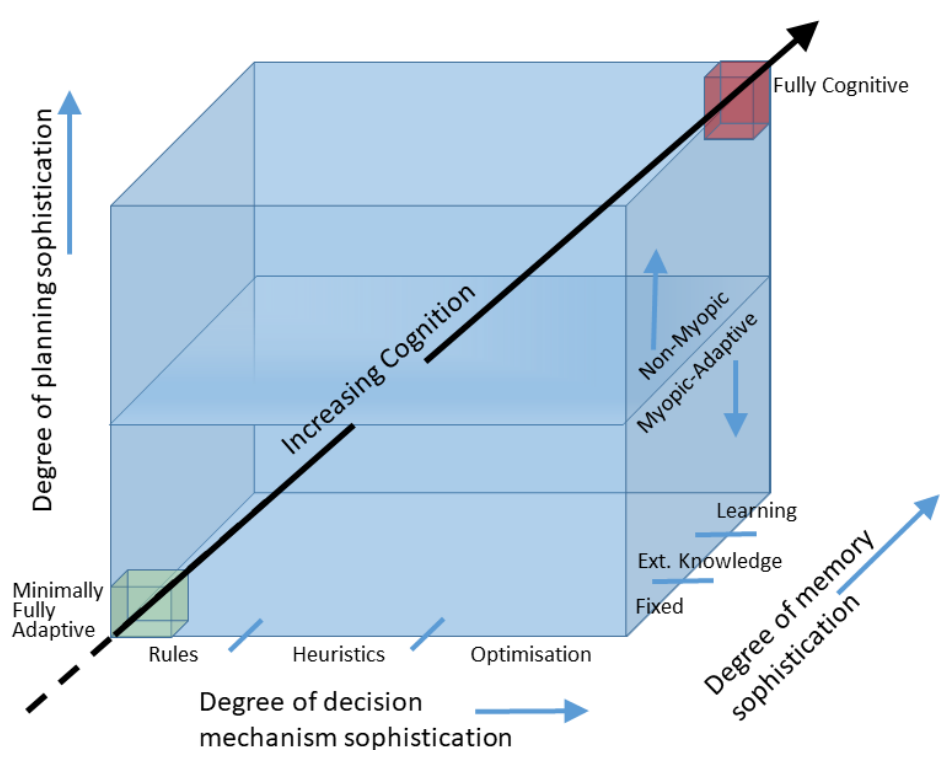

Fig. 4: 3-D Character of Radar Synthetic Cognition Space

All green (solid colour) region characteristics from Fig. 3, would appear on the dashed line extension to the bottom left of the cube, so are not contained in the 3D cognition space. Although these items could conceivably contain some of the characteristics employed to define the cube, such systems will either not contain all the necessary characteristics, or they will be implemented non-synthetically. 
Table 1 The dimensions of the 3D IR space

\begin{tabular}{|c|c|c|c|}
\hline & Planning (P) & $\begin{array}{l}\text { Memory/ } \\
\text { Learning (M) }\end{array}$ & Decision (D) \\
\hline 10 & Mission Level & $\begin{array}{l}\text { Understanding of } \\
\text { knowledge } \\
\text { New behaviour } \\
\text { creation }\end{array}$ & $\begin{array}{l}\text { Decisions based } \\
\text { on understanding } \\
\text { of learning/ } \\
\text { knowledge }\end{array}$ \\
\hline \multicolumn{4}{|c|}{ FULLY COGNITIVE } \\
\hline 9 & $\begin{array}{l}\text { Task timeframe, } \\
\text { multiple tasks }\end{array}$ & Abstraction & $\begin{array}{l}\text { Optimisation, } \\
\text { informed by } \\
\text { learning, } \\
\text { multi-parameter }\end{array}$ \\
\hline 8 & Task timeframe & Intention & $\begin{array}{l}\text { Optimisation, } \\
\text { informed by } \\
\text { learning }\end{array}$ \\
\hline 7 & $\begin{array}{l}\text { Non-myopic, } \\
\text { long timeframe, } \\
\text { multiple tasks }\end{array}$ & Identification & Optimisation \\
\hline 6 & $\begin{array}{l}\text { Non-myopic, } \\
\text { long timeframe }\end{array}$ & $\begin{array}{l}\text { Parameter } \\
\text { estimation, } \\
\text { multisensory, } \\
\text { external learning } \\
\text { agent }\end{array}$ & $\begin{array}{l}\text { Heuristics } \\
\text { informed by } \\
\text { learning, } \\
\text { multi-parameter }\end{array}$ \\
\hline 5 & $\begin{array}{l}\text { Non-myopic, } \\
\text { short timeframe, } \\
\text { multiple tasks }\end{array}$ & $\begin{array}{l}\text { Parameter } \\
\text { estimation, } \\
\text { multisensory }\end{array}$ & $\begin{array}{l}\text { Heuristics } \\
\text { informed by } \\
\text { learning }\end{array}$ \\
\hline 4 & $\begin{array}{l}\text { Non-myopic, } \\
\text { short timeframe }\end{array}$ & $\begin{array}{l}\text { Parameter } \\
\text { estimation }\end{array}$ & Fixed heuristics \\
\hline 3 & $\begin{array}{l}\text { Myopic, } \\
\text { multiple tasks }\end{array}$ & $\begin{array}{l}\text { Knowledge } \\
\text { updated by } \\
\text { external learning } \\
\text { agent }\end{array}$ & $\begin{array}{l}\text { Rules informed } \\
\text { by learning }\end{array}$ \\
\hline 2 & Myopic & $\begin{array}{l}\text { Fixed memory / } \\
\text { knowledge }\end{array}$ & Fixed rules \\
\hline \multicolumn{4}{|c|}{ MINIMALLY FULLY ADAPTIVE } \\
\hline 1 & $\begin{array}{l}\text { Non-synthetic } \\
\text { planning }\end{array}$ & $\begin{array}{l}\text { Non-synthetic } \\
\text { learning }\end{array}$ & $\begin{array}{l}\text { Non-synthetic } \\
\text { decision making } \\
\text { Or } \\
\text { Adaptive-on- } \\
\text { receive }\end{array}$ \\
\hline 0 & $\begin{array}{l}\text { No planning } \\
\text { necessary - all } \\
\text { planning outside } \\
\text { of system }\end{array}$ & No learning & $\begin{array}{l}\text { No decision } \\
\text { making required }\end{array}$ \\
\hline
\end{tabular}

The axes shown in Fig. 4 are divided into course increments in order to illustrate the general concept. Within the full classification system further subdivisions are defined, Table 1. This lists the categories relating to the three cube dimensions. These categories represent the level of ability of an IR along each dimension in the 3D cognition space. In each dimension the available space is divided into 11 partitions, ranging from 0 to 10 . The lowest levels represent the region of the dashed line in Fig. 4, outside of the 3D space. These are included so the categories are inclusive of Fig. 3. Level zero of all three dimensions represents the absence of behaviour expected to be seen in adaptive systems. The values of 1 for each dimension represents non-synthetic capability in the characteristic, which would be supplied by human involvement. These would encompass the Adaptive/Human-in-the-loop systems. In addition, the adaptive-on-receive systems are also defined at level 1.

Planning: In the planning dimension the cognition level rises with the timeframe over which the system is capable of achieving meaningful anticipation, and as a secondary factor, by the number of tasks considered. The lowest level considered to reside in the IR space is at level 2: Myopic. Myopic indicates that the system is capable of planning only a single step ahead. Data received and processed on the current epoch, allows decisions to be made and applied in the next. This level represents the adaptive radar case in which the system responds to current sensed conditions and reacts accordingly. Level 3 allows for multiple tasks, each being planned in a myopic way.

Non-myopic planning extends the adaptivity by allowing the system to select short term actions which appear to be suboptimal, but which provides, by some measure, a greater long term gain. Level 4 represents the non-myopic/short timeframe scenario, where the timeframe over which planning takes place is limited to a small number of coherent processing intervals. Multiple tasks are added in level 5. Level 6 is a longer timeframe non-myopic capability, of the order of many seconds. Level 8 extends the planning timeframe further to encompass a complete task, such as tracking a target until it is out of range. Level 9 adds multiple tasks. Finally, level 10 provides planning capability over mission duration timescales.

Memory/Learning: In this dimension the MFA capability, level 2, contains fixed memory content defined prior to the mission, and could be directly associated with known mission parameters. Level 3 allows for the knowledge base to be updated by an external learning agent. The higher levels embrace learning from the data provided by internal sensors, and potentially external sensors, with level 4 dealing with model parameter learning, such as the estimation of parameters for a tracking filter. Parameter estimation using multiple internal sensors applies at level 5, with level 6 extending this to include information from an external agent. Level 7 enables identification of scene components to be performed, and level 8 is capable of the evaluation of the 'intention' of the scene components. Level 9 takes the results from the learning exercise and abstracts the knowledge such that experience might be used to improve performance against a previously unseen scenario. Level 10 includes an understanding of the knowledge gained, and the creation of new behaviours to exploit that understanding.

Decision: Fixed rules are at the MFA level for the decision dimension. Decision processing using fixed rules uses data from the sensor to drive the selection of new actions, but critically, given identical sensor input, the selected action will be the same. Level 3 adds the ability for the rules to be modified by learned knowledge, such that performance improves over time. Level 4 uses fixed heuristic decision making, while level 5 adds learned modification to the heuristics. Multiple parameter heuristics are introduced at level 6. Level 7 employs optimisation techniques. Level 8 allows optimisation modified by learning, and level 9 adds multiple parameters to the optimisation. Level 10 decisions are based on an 'understanding' of learned knowledge.

For each case Level 10 indicates capabilities beyond the 3D cognition space being considered, allowing for mission timeframe planning, an understanding of the meaning of learned information, and the ability to reason based on such understanding.

The three values allocated to a system from the dimensions of the cognition space cube, when taken together, provide a direct indication of the level of cognition. The three values are reported as a 3-tuple, for example, the result might 
be [4:6:5], representing the individual values for [planning:memory:decision]. The order is arbitrary, but fixed. No hierarchical significance is to be placed on the ordering of the values.

A three value system is proposed instead of a single numerical designation for the cognitive ability of IR. This is because of the challenge in creating a system devoid of ambiguity and discontinuities. The 3-tuple values may be combined, for example using the minimum values, the means, and the variance, but currently no satisfactory solution is available.

Table 2 Analysis of reported capability

\begin{tabular}{|l|l|l|}
\hline No. & Description & $\begin{array}{l}\text { Level } \\
\text { [P:M:D] }\end{array}$ \\
\hline 1 & $\begin{array}{l}\text { ATC [76] } \\
\text { Non-synthetic input on all dimensions. }\end{array}$ & {$[1: 1: 1]$} \\
\hline 2 & $\begin{array}{l}\text { CREW [66] } \\
\text { PRF / Number of pulses adjustment for } \\
\text { target tracking. } \\
\text { [Single-task myopic/Internal estimation of } \\
\text { parameters/Optimisation] }\end{array}$ & {$[2: 4: 7]$} \\
\hline 3 & $\begin{array}{l}\text { Waveform design [25] } \\
\text { Learning of noise covariance matrix. } \\
\text { Design of waveform. } \\
{[\text { Single-task myopic/Internal estimation of }} \\
\text { parameters/Fixed rule decision] }\end{array}$ & {$[2: 4: 2]$} \\
\hline 4 & $\begin{array}{l}\text { HF radar [68] } \\
\text { Adaptive. Ionospheric and spectral } \\
\text { occupancy estimation. Rule based } \\
\text { knowledge engine based on long term } \\
\text { experience, and updated offline by new } \\
\text { experience. } \\
{[\text { Multi-task myopic/Internal estimation of }} \\
\text { parameters/Fixed rule decision] }\end{array}$ & {$[3: 4: 2]$} \\
\hline
\end{tabular}

Example algorithms and systems have been assessed within the proposed classification schemed and are shown in Table 2. The first example is the ATC system having a human as part of its assessment as a CR. Level 1 in all dimensions is chosen due to the non-synthetic components in the architecture. This is followed by the leading CR experimental setup, CREW. This system has been shown to adapt radar parameters in real-time in order to maintain a specified SNR on a target, while maintaining the target in the unambiguous Doppler space, and avoiding stationary clutter [66]. This work is advanced in the learning and decision-making dimensions, but is limited to adaptive planning. In the domain of waveform design the work from Guerci [25] is found to be equally cognitive in the planning and learning dimensions as the CREW experimentation, but lower in the decision making dimension. This being due an optimisation process not being necessary in this case. Finally, HF radar cognition is assessed as being at similar levels to the waveform design case, but it should be remembered that this work was carried out in 1986.When using the classification of IR for comparing competing solutions, we should be mindful of increased complexity with little benefit. If a lower graded system performs equally well in the context of the application, the higher graded system offers no advantage. This is shown by case 3, Table 2, in terms of the level of decision making being low, but the correct level for the application.

\section{Conclusion}

The purpose of this article is twofold, to introduce a framework for classifying IR, and secondly to review the CR research domain, and promote discussion into the nature of cognition as it is be applied in radar system, and specifically, how IR should be classified.

A framework is proposed to allow the classification of cognitive elements within IR systems. It should be understood that this is a fast evolving, very fluid domain in which new research may disrupt our current understanding, resulting in a classification scheme which is likely to evolve over time.

Providing a scale measure of systems' ability is not new. Precedent exists, for example the field of autonomous vehicles, which employ such scales to represent the degree of autonomy exhibited in vehicles. IR research can still be regarded as being in its infancy, so defining a classification scheme for IR at this early stage of research will avoid the proliferation of competing classification systems, which can be seen to have occurred in the autonomous vehicles discipline, with the resulting ambiguity that brings.

The realisation that the degree of radar cognition should be regarded as a scale rather than a binary attribute is fundamental to defining this classification system. It should be noted that not all systems need to be cognitive at the highest levels, only sufficient cognition need be incorporated in any system to achieve the desired outcome, although future proofing of systems by the addition of extra levels of cognition could offer long term benefits in terms of system longevity.

The proposed tools should be applied to all stakeholder functions, such that identical understanding of capability is seen in classification, specification, procurement and acceptance of IR.

The existing literature does not address the classification of IR. The scheme presented in this article begins the process of addressing that requirement.

\section{Acknowledgements}

The authors acknowledge the support of EPSRC and the Defence Science and Technology Laboratory (Dstl).

\section{References}

[1] Haykin, S.: 'Radar Vision', IEEE Int. Radar Conference, Arlington, VA, 1990, pp. 585-588

[2] Haykin, S.: 'Adaptive radar: evolution to cognitive radar', IEEE International Symposium on Phased Array Systems and Technology, Boston, MA, 2003, p. 613

[3] Haykin, S.: 'Cognitive Radar [A way of the future]', IEEE Signal Processing Magazine, 2006, 23, (January), pp. 3040.

[4] Martone, A.F.: 'Cognitive Radar Demystified', URSI Radio Science Bulletin, 2014, 2014, (350), pp. 10-22.

[5] UK Department for Culture Media \& Sport: 'The UK Spectrum Strategy Delivering the best value from spectrum for the UK', 2014 
[6] UK Department for Culture Media \& Sport: 'Enabling UK growth - Releasing public spectrum Making $500 \mathrm{MHz}$ of spectrum available by $2020^{\prime}, 2011$

[7] Federal Communications Commission: 'Auction of Advanced Wireless Services (Aws-3) Licenses Closes', https://apps.fcc.gov/edocs_public/attachmatch/DA-15131A1.pdf, accessed June 2018

[8] Science and Technology Committee: 'https://publications.parliament.uk/pa/cm201011/cmselect /cmsctech/619/61913.htm Annex 1: Technology Readiness Levels', 2011

[9] EDA: 'Working Paper - Best Practice Guide for UMS Handling', 2012

[10] National Institute Of Standards And Technology: 'Autonomy Levels For Unmanned Systems (ALFUS) framework', 2005

[11] Arrabales, R., Ledezma, A., Sanchis, A.: 'ConsScale', Journal of Consciousness Studies, 2010, 17, (3-4), pp. 131-164.

[12] Fuster, J.: Cortex and Mind: Unifying Cognition, Oxford University Press, New York, 2003

[13] Haykin, S., Xue, Y., Davidson, T.N.: 'Optimal Waveform Design For Cognitive Radar', Asilomar Conference on Signals, Systems and Computers, Pacific Grove, 2008, pp 3-7

[14] Haykin, S.: 'Cognitive Radar Networks', 1st IEEE International Workshop on Computational Advances in Multi-Sensor Adaptive Processing., Puerto Vallarta, Mexico, 2005

[15] Capraro, G., Baldygo, W., Day, R., Perretta, J., Wicks, M.: 'Autonomous Intelligent Radar System (AIRS) for multi-sensor radars', IEEE CAMSAP 2005 - First International Workshop on Computational Advances in Multi-Sensor Adaptive Processing, Puerto Vallarta, Mexico, 2005, pp. 16-19

[16] Haykin, S., Zia, A., Arasaratnam, I., Xue, Y.: 'Cognitive tracking radar', IEEE Radar Conference, Washington, DC, 2010, pp. 1467-1470

[17] Haykin, S., Zia, A., Xue, Y., Arasaratnam, I.: 'Control theoretic approach to tracking radar: First step towards cognition', Digital Signal Processing, 2011, 21, (5), pp. 576-585.

[18] Haykin, S., Fatemi, M., Setoodeh, P., Xue, Y.: 'Cognitive Control', Proc. IEEE, 2012, 100, (12), pp. 3156-3169.

[19] Fatemi, M., Haykin, S.: 'Cognitive Control : Theory and Application', IEEE Access, 2014, 2, pp. 698-710.

[20] Haykin, S.: 'Cognitive Radar Networks', Fourth IEEE Workshop on Sensor Array and Multichannel Processing, Waltham, Massachusetts, 2006, pp. 9-30

[21] Haykin, S.: 'Cognitive Networks : Radar, Radio, and Control for New Generation of Engineered Complex Networks', IEEE Radar Conference, Ottawa, ON, 2013

[22] Guerci, J.R., Pillai, S.U.: 'Adaptive transmission radar: the next "wave"?', Proceedings of the IEEE National Aerospace and Electronics Conference, Dayton, $\mathrm{OH}$, 2000, pp. 779-786

[23] Garren, D.A., Odom, A.C., Osborn, M.K., Goldstein, J.S., Pillai, S.U., Guerci, J.R.: 'Full-polarization matchedillumination for target detection and identification', IEEE Transactions on Aerospace and Electronic Systems, 2002, 38, (3), pp. 824-837.

[24] Guerci, J.R., Baranoski, E.J.: 'Knowledge-Aided Adaptive Radar at DARPA', IEEE Signal Processing Magazine, 2006, 23, (1), pp. 41-50.
[25] Guerci, J.R.: 'Cognitive Radar: The Next Radar Wave?', Microwave Journal, 2011, 54, (1), pp. 22-36.

[26] Guerci, J.R.: 'Cognitive radar: A knowledge-aided fully adaptive approach', IEEE Radar Conference, Washington, DC, 2010, pp. 1365-1370

[27] Griffin, D.R., Friend, J.H., Webster, F.A.: 'Target Discrimination by the Echolocation of Bats', Journal of Experimental Zoology, 1965, 158, (2), pp. 155-168.

[28] von Helversen, D., von Helversen, O.: 'Object recognition by echolocation: a nectar-feeding bat exploiting the flowers of a rain forest vine.', Journal of comparative physiology. A, Neuroethology, sensory, neural, and behavioral physiology, 2003, 189, (5), pp. 327-36.

[29] Vespe, M., Jones, G., Baker, C.J.: 'Lessons for Radar [waveform diversity in echo locating mammals]', IEEE Signal Processing Magazine, 2009, 26, (1), pp. 65-75.

[30] Baker, C.J., Woodbridge, K., Holderied, M.W., Balleri, a., Griffiths, H.D.: 'Analysis of acoustic echoes from a bat-pollinated plant species: insight into strategies for radar and sonar target classification', IET Radar, Sonar \& Navigation, 2012, 6, (6), pp. 536-544.

[31] Baker, C.J., Smith, G.E., Balleri, A., Holderied, M., Griffiths, H.D.: 'Biomimetic Echolocation With Application to Radar and Sonar Sensing', Proc. IEEE, 2014, 102, (4), pp. 447-458.

[32] Au, W.W.L., Simmons, J.A.: 'Echolocation in dolphins and bats', Physics Today, 2007, 60, (9), pp. 40-45.

[33] Wisniewska, D.M., Ratcliffe, J.M., Beedholm, K., et al.: 'Range-dependent flexibility in the acoustic field of view of echolocating porpoises ( Phocoena phocoena )', ELife, 4. https://doi.org/10.7554/eLife.05651, 2015, (March).

[34] Thaler, L., Reich, G.M., Zhang, X., et al.: 'Mouth-clicks used by blind expert human echolocators - signal description and model based signal synthesis', PLoS Computational Biology, 2017, 13, (8), pp 1-17.

[35] Thaler, L., De Vos, R., Kish, D., Antoniou, M., Baker, C., Hornikx, M.: 'Human echolocators adjust loudness and number of clicks for detection of reflectors at various azimuth angles.', Proc. R. Soc. B, 2018, 285, (1873).

[36] Balleri, A., Leighton, T.G.: 'Editorial: Biologicallyinspired radar and sonar systems', IET Radar, Sonar \& Navigation, 2012, 6, (6), pp. 507-509.

[37] Wicks, M.C.: 'A Brief History of Waveform Diversity', IEEE Radar Conference, Passadena, 2009

[38] Wicks, M.: 'Spectrum Crowding and Cognitive Radar', 2nd International Workshop on Cognitive Information Processing (CIP), Elba Island, 2010, pp. 452-457

[39] Griffiths, H., Blunt, S., Cohen, L., Savy, L.: 'Challenge problems in spectrum engineering and waveform diversity', IEEE Radar Conference, Ottawa, 2013

[40] Griffiths, H., Cohen, L., Watts, S., et al.: 'Radar spectrum engineering and management: Technical and regulatory issues', Proc. IEEE, 2015, 103, (1), pp. 85-102.

[41] DeLong, D., Hofstetter, E.: 'On the design of optimum radar waveforms for clutter rejection', IEEE Transactions on Information Theory, 1967, 13, (3), pp. 454-463.

[42] DeLong, D., Hofstetter, E.: 'The Design of ClutterResistant Radar Waveforms with Limited Dynamic Range', IEEE Transactions on Information Theory, 1969, 15, (3), pp. 376-385.

[43] Stinco, P., Greco, M.S., Gini, F.: 'Spectrum sensing and sharing for cognitive radars', IET Radar, Sonar \& Navigation, 2016, 10, pp. 595-602.

[44] Goodman, N.A.: 'Closed-loop radar with adaptively 
matched waveforms', 2007 International Conference on Electromagnetics in Advanced Applications, ICEAA'07, Torino, 2007, pp. 468-471

[45] La Manna, M., Stinco, P., Greco, M., Gini, F.: 'Design of a cognitive radar for operation in spectrally dense environments', IEEE Radar Conference, Ottawa, 2013

[46] La Manna, M., Stinco, P., Greeo, M., Gini, F.: 'Cognitive techniques for a wideband phased array radar', IEEE International Symposium on Phased Array Systems and Technology, Boston, Massachusetts, 2013, pp. 389-393

[47] Aubry, A., De Maio, A., Piezzo, M., Farina, A.: 'Radar waveform design in a spectrally crowded environment via nonconvex quadratic optimization', IEEE Transactions on Aerospace and Electronic Systems, 2014, 50, (2), pp. 1138-1152.

[48] Aubry, A., Carotenuto, V., Maio, A. De, Iommelli, S.: 'Cognitive Radar Waveform Design for Spectral Compatibility', 2016 Sensor Signal Processing for Defence (SSPD), Edinburgh, 2016

[49] Aubry, A., De Maio, A., Piezzo, M., Farina, A., Wicks, M.: 'Cognitive design of the receive filter and transmitted phase code in reverberating environment', IET Radar, Sonar \& Navigation, 2012, 6, (9), pp. 822-833.

[50] Blunt, S.D., Mokole, E.L.: 'An Overview of Radar Waveform Diversity', IEEE Aerospace \& Electronic System Magazine, 2016, 31, (11), pp. 2-40.

[51] Gjessing, D.: 'Adaptive Techniques for Radar Detection and Identification of Objects in an ' Ocean Environment', EEE Journal of Oceanic Engineering, 1981, 6, (1), pp. 517.

[52] Gjessing, D.T., Saebboe, J., Helleren, O.E.: 'Recognition of Targets by Linear and Non-Linear ( Delta K ) Processing of Multi Frequency Data', RTO SET Symposium on 'Target Identification and Recognition Using RF Systems', Oslo, 2004

[53] Soldani, F., Alabaster, C.M.: 'The benefits of matched illumination for radar detection of ground based targets', International Waveform Diversity and Design Conference, Pisa, 2007, pp. 23-27

[54] Romero, R.A., Bae, J., Goodman, N.A.: 'Theory and application of SNR and mutual information matched illumination waveforms', IEEE Transactions on Aerospace and Electronic Systems, 2011, 47, (2), pp. 912927.

[55] Romero, R.A., Goodman, N.A.: 'Waveform design in signal-dependent interference and application to target recognition with multiple transmissions', IET Radar, Sonar \& Navigation, 2009, 3, (4), pp. 328-340.

[56] Goodman, N.A., Venkata, P.R., Neifeld, M.A.: 'Adaptive waveform design and sequential hypothesis testing for target recognition with active sensors', IEEE Journal on Selected Topics in Signal Processing, 2007, 1, (1), pp. 105-113.

[57] Smits, F., Huizing, A., Rossum, W. Van, Hiemstra, P.: 'A Cognitive Radar Network: Architecture and Application to Multiplatform Radar Management', European Radar Conference, Amsterdam, 2008, pp. 312-315

[58] Nijsure, Y., Chen, Y., Boussakta, S., Yuen, C., Chew, Y.H., Ding, Z.: 'Novel System Architecture and Waveform Design for Cognitive Radar Radio Networks', IEEE Transactions on Vehicular Technology, 2012, 61, (8), pp. 3630-3642.

[59] Charlish, A.B.: 'Tasking networked multi-function radar systems for active tracking', 14th International Radar Symposium (IRS), Dresden, 2013, pp. 367-374
[60] Kershaw, D.J., Evans, R.J.: 'Optimal Waveform Selection for Tracking Systems', IEEE Transactions on Information Theory, 1994, 40, (5), pp. 1536-1550.

[61] Romero, R., Goodman, N.: 'Cognitive Radar Network : Cooperative Adaptive Beamsteering for Integrated Search-and-Track Application', IEEE Transactions on Aerospace and Electronic Systems, 2013, 49, (2), pp. 915931.

[62] Kreucher, C.M., Hero, A.O., Kastella, K.D., Morelande, M.R.: 'An information-based approach to sensor management in large dynamic networks', Proceedings of the IEEE, 2007, 95, (5), pp. 978-999.

[63] Kreucher, C., Hero, A.O., Kastella, K.: 'A comparison of task driven and information driven sensor management for target tracking', Proceedings of the 44th IEEE Conference on Decision and Control, and the European Control Conference, CDC-ECC, Seville, 2005, pp. 4004-4009

[64] Sherwani, H., Griffiths, H.D.: 'Tracking parameter control in multifunction radar network incorporating information sharing', FUSION 2016 - 19th International Conference on Information Fusion, Heidelberg, 2016

[65] Bell, K.L., Baker, C.J., Smith, G.E., Johnson, J.T., Rangaswamy, M.: 'Cognitive Radar Framework for Target Detection and Tracking', IEEE Journal on Selected Topics in Signal Processing, 2015, 9, (8), pp. 1427-1439.

[66] Smith, G.E., Cammenga, Z., Mitchell, A., et al. 'Experiments with cognitive radar', IEEE Aerospace and Electronic Systems Magazine, 2016, 31, (12), pp. 34-46.

[67] Oechslin, R., Smith, G.E., Aulenbacher, U., Rech, K., Hinrichsen, S., Bell, K.L.: 'Cognitive Radar Testbed Development Special Session on Cognitive Radar', Asilomar Conference on Signals, Systems and Computers, Pacific Grove, 2016

[68] Anderson, S.J.: 'Remote Sensing with the JINDALEE Skywave Radar', IEEE Journal of Oceanic Engineering, 1986, 11, (2), pp. 158-163.

[69] Lu, K., Chen, X.: 'Cognitive over-the-horizon radar', Proceedings of 2011 CIE International Conference on Radar,2011, pp. 993-996

[70] Saverino, A.L., Capria, A., Berizzi, F., Dalle Mese, E. 'Cognitive adaptive waveform technique for HF skywave radar', 2010 2nd International Workshop on Cognitive Information Processing, CIP2010, Elba Island, 2010, pp. 247-252

[71] Holdsworth, D.A.: 'An over-the-horizon radar performance assessment module for use in cognitive radar', IET International Conference on Radar Systems, Glasgow, 2012, pp. 34-34

[72] Wicks, M.C.: 'Radar the Next Generation - Sensors as Robots', International Radar Conference, Adelaide, 2003, pp. 7-14

[73] Greenspan, M.: 'Potential Pitfalls of Cognitive Radars', IEEE Radar Conference, Cincinnati, Ohio, 2014, pp. $1288-1290$

[74] Bruggenwirth, S.: 'Design and implementation of a threelayer cognitive radar architecture', Conference Record Asilomar Conference on Signals, Systems and Computers, Pacific Grove, 2017, pp. 929-933

[75] IEEE Standards Association: 'IEEE P686 Standard radar definitions', 2017

[76] Baker, C., Smith, G.: 'The Case for Cognition and Radar Sensing', NATO Lecture Series EN-SET-216,2015

[77] Farina, A.: 'Introduction To Radar Signal \& Data Processing : The Opportunity', RTO-EN-SET-063,2006, pp. 28-29 
[78] Inggs, M.: 'Passive Coherent Location as Cognitive Radar', IEEE Aerospace and Electronic Systems Magazine, 2010, 25, (5), pp. 12-17.

[79] Vernon, D., Metta, G., Sandini, G.: 'A Survey of Artificial Cognitive Systems : Implications for the Autonomous Development of Mental Capabilities in Computational Agents', IEEE Transactions on Evolutionary Computation, 2007, 11, (2), pp. 151-180.

[80] Charlish, A., Hoffmann, F.: 'Anticipation in Cognitive Radar using Stochastic Control', IEEE Int. Radar Conference, Arlington, VA, 2015, pp. 1692-1697

[81] Horne, C.P., Ritchie, M., Griffiths, H.D., Hoffmann, F., Charlish, A.: 'Experimental Validation of Cognitive Radar Anticipation using Stochastic Control', Asilomar Conference on Signals, Systems and Computers, Pacific Grove, 2016

[82] Tweedale, J.W.: 'A Review of Cognitive Decision-making within Future Mission Systems', Procedia Computer Science, 2014, 35, pp. 1043-1052.

[83] Kyllonen, P., Zu, J.: 'Use of Response Time for Measuring Cognitive Ability', Journal of Intelligence, 2016, 4, (4), p. 14. 